\title{
Design of SaaS Layer Cloud Platform for Rapid Design of Series Products
}

\author{
Wuxue Jiang ${ }^{1}$, Dukun Ding ${ }^{2}$, Yuqiang Chen ${ }^{1}$, Wenliang Cao ${ }^{1}$, Yan Liang ${ }^{3}$ \\ 1.Department of Computer Engineering, Dongguan Polytechnic, Dongguan, 523808, China
}

2. Department of Mechetronics Engineering, Dongguan Polytechnic, Dongguan, 523808, China

3.Department of Computer Engineering, Maoming Polytechnic, Maoming, 525000, China

Keywords: Rapid design of product, SaaS, Cloud platform, Service-oriented integration, J2EE.

\begin{abstract}
Correlation theories for rapid design of series products under SaaS model was illustrated and system instances were established in this thesis in allusion to the improvement of informatization level of modern enterprises, as well as the improvement of the efficiency and quality of rapid design of product. Besides, the main technologies of multi-tenant design, data expansion design and flexibility configuration design in SaaS service mode system were studied, and a system prototype was achieved by applying the above key technologies. It is different from transitional J2EE system structure on account of that it systematically made use of Struts frame to realize presentation layer and control layer, added DAO Factory logic module to link different data, instantiated the object and achieve specific operation.
\end{abstract}

\section{Introduction}

Rapid design is also called quick response design or agile design, and it was proposed and developed under the situation of the need of current market to product diversification and transient behavior. Rapid design of product is a new kind of philosophy, and the main purpose of it is to shorten the design cycle of the product, improve the design quality of the product, and improve the company's quick response ability to the market when compared with computer integrated manufacturing (CIM), lean project (LP), concurrent engineering (CE), and agile manufacturing (AM). As engineering concept, the typical examples of product platform since 90s of last century included: Kodak Company successively developed four kinds of camera series products in allusion to different market segments on the basis of the same platform; different types of walkmans produced by Sony shared similar recording mechanism; Xerox produced different types of duplicators by adopting modularization platform in order to meet the demands of different users.

At present, enterprises make product design mainly through amending existing accessories, which can effectively shorten the design cycle of the product, and allow the company to develop in fierce competition [1]. The main method adopted by rapid design currently is to complete the design work of the product by modifying or improving the existing product or design examples, namely, to complete the design of new product rapidly with high quality and satisfy the need of clients by making variant design to the structure parameter and other properties of the product on the basis of keeping the function and principle of the product unchanged [2].

\section{SaaS Layer Cloud Services}

Cloud computing was developed from parallel processing, distributed processing and grid computing, and it transfers the data processing in personal computer or server to supercomputing group in internet which consists of thousands of general industrial standard server, so as to obtain high-speed data handling capacity.

SaaS providers can set up all network infrastructure and soft hardware operation platform needed by informatization of enterprises, and they are responsible for a series of work including previous implementation and later maintenance, by this way, companies can use information systems through internet without the need of purchasing soft hardware, constructing machine room, or recruiting IT 
personnel. It is just like using water after turning on the water tap, and the companies can rent software maintenance from SaaS providers in accordance with its needs.

$\mathrm{SaaS}$ is a kind of software layout model, and its application is designed specially for network delivery, which is convenient for users to deposit, deploy and insert through the internet [3]. The price of SaaS application software is generally all-inclusive fee, which includes software license fee of general application software, maintenance cost and technical support fee of software, and uniform them into monthly lease charges of each user. SaaS is the best way for small and mediumsized enterprises to realize informatization by making use of advanced technology. While, SaaS is definitely not just suitable for small and medium-sized enterprises, but all the enterprises with different scales can make a profit from SaaS.

\section{Correlation Technique for Rapid Design of Products}

Systematic framework of SaaS model. It made a research on software system architecture under SaaS model and designed database by deeply analyzing the difference and relation between SaaS model and ASP model, and centering on multi-tenant character of SaaS model.

Enterprise information service integration and software combination technique. SOI is the integration made by WEB service under SOA circumstances, and it can solve integration and interoperability problems by applying WEB service systematically. It inherited excellent technologies of EAI, message bus, process integration, data integration, etc., and used standard integration which was the same as Web service, besides, it translated a number of associative functions of existing system into service to make integration by providing a systematic frame used for constructing, deploying and administrating the integration, which simplified the integration process of the system.

The design of dynamic service selection for multi-tenant application and its deployment strategy. In order to solve the difficulty of multi-tenant application in performance customization, it needed to convert it into selection problem of dynamic state service driven by SLA to solve under the condition that each logical service corresponded to multiple service instances with different nonfunctional properties, then it realized the partition of tenant type by clustering method, after that, the service selection driven by SLA would be achieved by making use of genetic algorithm based on real time load data.

System development and the realization of examples. It established the frame structure of rapid design of series products under SaaS model; achieved SaaS framework of software on the basis of making research on software system architecture of SaaS model and the design method of database; secondly, the development of SaaS software application program layer, rapid design software and rapid quotation of series products have been realized; conducted the establishment of gene pool of rapid design of product, the development of design module of parameter and model driven module, the development and verification of technique information statistics module based on the theoretical basis of rapid design of genetics products; finally, it proceeded component encapsulation and debugging movement by facing to typical manufacturing industry [4]. The comparison relation between the design platform rental models of traditional products and the design platform rental model of products on SaaS layer was shown in Fig.1. 


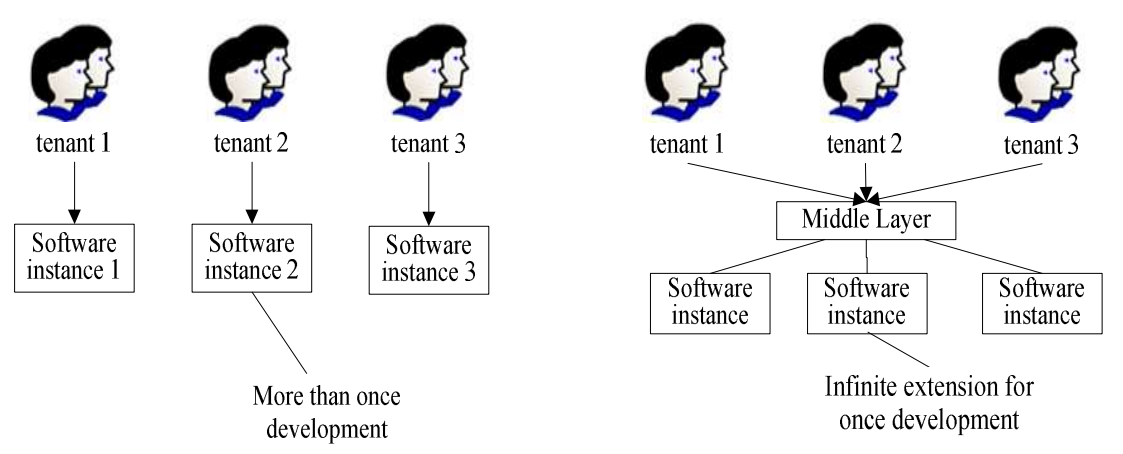

Fig. 1: Comparison of the two pattern between traditional and rental

\section{Design Cases}

J2EE is a standard system structure, and provides multilevel system structure which is centered on application server and based on component [5]. Such kind of multilevel distributed system structure can make logical functions be divided into different components, moreover, the components can be distributed on different machines in accordance with the distribution of the layer they were on, and the system general architecture is shown in Fig.2.

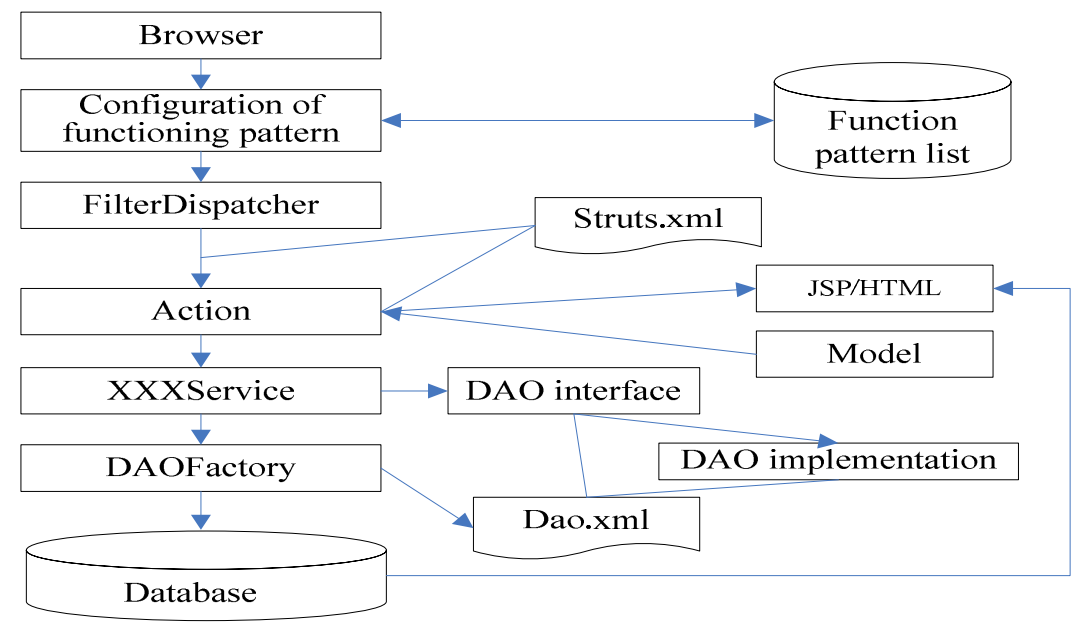

Fig. 2: Product design cloud platform system architecture chart

Client layer: J2EE application can be based on Web browser, or is not based on it. In its application based on Web browser, the browser would operate on client layer and can download HTML page from Web browser. In its application which is not based on Web browser, the operation of client layer would not visit Entersprise Beans through web, but, the client layer was used for interacting with users and showing information to users.

Web layer: Web consists of JSP page and Servlets. It can send the input of users to business layer and make it be processed by Entersprise Beans.

Business layer: it is used for solving business logic, and there will be Entersprise Beans for execution. One Entersprise Beans can receive data from client end to dispose, and then it will sent back the data to client end after completing the disposition of relevant data by sending it to EIS layer. The interlayer can be constructed by business layer and Web layer together.

EIS layer: it includes database system, transaction processing system and enterprise resource planning system and other enterprise information services.

Systematic architecture design: the original system adopted the combination of $\mathrm{J} 2 \mathrm{EE}+$ Struts + Hibernate frame, and it was with certain advantage in aspect of usage, however, it was unfavorable for the construction of service mode system of platform, meanwhile, Hibernate frame lowered the flexibility of user configuration. This thesis remolded traditional frame, made use of 
Struts frame to realize presentation layer and control layer, built and connected different databases by adding DAOFactory logic, instantiated the object and allowed specific operation to be realized.

Tenant can make system operation through browser. When users logged in at the first time, they can make functional pattern configuration, upload various role functions of tenant in system, besides, users can make optional configuration to specific function according to their needs again after entering into actual interface [6]. When delivering data, users can search the corresponding action through Struts.xml file to make logical disposition, after that, the system in the article would separate the actual business process from Action and make practical disposition to the function by calling business logic module, in this way, the need of system function regulation can be realized through making configuration to dao.xml. Business logic module is actually a DAOFactory module which can achieve the connection of database and various specific operations.

\section{Conclusion}

This thesis expounded the correlation theory of rapid design of series products under SaaS mode, and established systematical examples. In addition, the advantages and disadvantages of SaaS service mode, the difference and relation between SaaS service mode and ASP were analyzed in detail; the seven-layer system framework structure and the corresponding database design scheme of SaaS mode software system were proposed on the basis of the maturity degree of the third stage. Furthermore, it made a research on the key technologies of SaaS service mode system, including multi-tenant design, data expansion design and flexibility configuration design etc., and one system prototype was realized by making use of the above key technologies.

\section{Acknowledgements}

This work was financially supported by Guangdong province science and technology plan projects(No.2014A010103002), and by Guangdong Province University Outstanding Young Teachers' Training Program(No.Yq2013201), and by production-study-research cooperation projects in dongguan city (No.2014509102211), and by Production-study-research cooperation project in guangdong province (No.2013B011301003), and by the project of social science and technology of Dongguan City in 2013- "Research on key technology of product public opinion mining based on high performance computing" (No.2013108101045), and by the fund project of Dongguan Polytechnic (No.2014a06, No.2014a07, No.2014d16), and by the Key Teaching Reform Project of Dongguan Polytechnic (JGXM2014020).

\section{References}

[1] Wei-Wen Wu. Developing an explorative model for SaaS adoption. Expert System with Applications, 38(12), pp. 15057-15064, 2011.

[2] Björn Johansson, Pedro Ruivo. Exploring Factors for Adopting ERP as SaaS. Procedia Technology, 9, pp. 94-99, 2013.

[3] David Concha, Javier Espadas, David Romero, Arturo Molina. The e-HUB evolution: From a Custom Software Architecture to a Software-as-a-Service implementation. Computers in Industry, 61(2), pp.145-151, 2010.

[4] Alexander Benlian, Thomas Hess. Opportunities and risks of software-as-a-service: Findings from a survey of IT executives. Decision Support Systems, 52(1), pp. 232-246, 2011.

[5] Sangjae Lee, Sung Bum Park, Gyoo Gun Lim. Using balanced scorecards for the evaluation of "Software-as-a-service”. Information \& Management, 50(7), pp. 553-561, 2013.

[6] CJ Radford. Challenges and solutions protecting data within Amazon Web Services. Network Security,2014( 6), pp.5-8, 2014. 
[7] Yinling Jiang, Ji Qi, Zixuan Gao, 2014, The Neural Network Sliding Mode Controller Based on Multiple Model for Robotic Manipulators, Journal of applied science and engineering innovation, Vol.1, No.6, pp,370-375.

[8] Xiuqiong Hu, Ying Wang,2014, A New Voltage Stability Control Model Considering Active Power Constraints on Weak Branches, Journal of applied science and engineering innovation, Vol.1, No.4, pp,268-274. 